\title{
Theranostic Agents in Musculoskeletal Disorders
}

\author{
Sanaz Katal, MD, MPH ${ }^{a}$, Antonio Maldonado, MD ${ }^{\mathrm{b}}$, Javier Carrascoso, MDc \\ Majid Assadi, MD, FASNC ${ }^{d}$, Ali Gholamrezanezhad, MD, FEBNM, DABR ${ }^{e, *}$
}

\section{KEYWORDS}

- Theranostics • Rheumatoid arthritis • Osteoarthrosis • Multiple myeloma • Sarcoma

- Glucocorticoid-loaded liposomes • Macrophage imaging • Radiolabeled anti-CD38 antibodies

\section{KEY POINTS}

- Theranostic approaches combine a single agent's therapeutic and diagnostic characteristics to achieve a targeted molecular therapy, with little or no toxicity to normal tissues.

- Selective theranostic agents have shown potential for targeted imaging and therapy in a variety of musculoskeletal illnesses.

- Some of these strategies include macrophage-related tools in inflammatory arthritis, chemokine receptor agents in multiple myeloma, and endothelial antibodies (TEM-1) in sarcoma.

\section{RHEUMATOID ARTHRITIS}

Rheumatoid arthritis (RA) is a chronic autoimmune joint and soft tissue disease that affects approximately $0.5 \%$ to $1 \%$ of the world population. ${ }^{1}$ It is a systemic inflammatory disease defined by polyarticular synovitis, causing irreversible joint damage and deformity, if not treated. Early diagnosis and treatment of RA remain a major clinical challenge. No definite curative treatments are available to date and the currently available drugs for RA only delay the disease progression. Symptomatic therapy and inflammation reduction with glucocorticoids (GCs), methotrexate (MTX), nonsteroidal anti-inflammatory drugs, azathioprine, tumor necrosis factor- $\alpha$ blockers, and minocycline is applied for symptomatic control of the disease.

The available medications used in the management of RA cause various short- and long-term side effects. Thus, there is a great need for biocompatible novel therapies with high targeted efficiency and minimal unwanted toxicity. Selective theranostic agents have shown potential for targeted imaging and therapy for inflamed joints, with the least collateral toxicity to the healthy tissues. ${ }^{2,3}$

\section{Glucocorticoid-Loaded Liposomes in Rheumatoid Arthritis}

As a well-documented anti-inflammatory drug, GCs are being used widely in the management of RA. However, they pose many side effects. To reduce the systemic toxicity of GCs and to enhance the RA treatment, encapsulation of GCs into long-circulating liposomes (LCLs) has been proposed. LCLs preferentially accumulate in the inflamed tissue, allowing selective targeting to diseased versus healthy tissue.

In-depth knowledge of the in vivo behavior of LCLs with molecular imaging may offer a great chance to monitor the drug biodistribution within the host body. Besides, it will also allow clinicians to select the best candidates for such therapies. This might be of great importance, because the kinetic properties of LCLs vary widely among different patients and also between different

\footnotetext{
a Department of Nuclear Medicine, Kowsar Hospital, Shiraz, Iran; ${ }^{b}$ Department of Nuclear Medicine, Quironsalud Madrid University Hospital, 28223 Pozuelo de Alarcón, Madrid, Spain; ' Department of Radiology, Quironsalud Madrid University Hospital, 28223 Pozuelo de Alarcón, Madrid, Spain; d Department of Molecular Imaging and Radionuclide Therapy (MIRT), The Persian Gulf Nuclear Medicine Research Center, Bushehr University of Medical Sciences, Bushehr, Iran; ${ }^{e}$ Department of Diagnostic Radiology, Keck School of Medicine, University of Southern California (USC), Los Angeles, CA, USA

* Corresponding author.

E-mail addresses: A.gholamrezanezhad@yahoo.com; ali.gholamrezanezhad@med.usc.edu
} 